\title{
Changes in the Energy Spectrum of Anomalous Oxygen During 1977-1985
}

\author{
A. C. Cummings and E. C. Stone \\ California Institute of Technology, Pasadena \\ W. R. WEBBER \\ University of New Hampshire, Durham
}

\begin{abstract}
We have used data from the cosmic ray experiments on the Voyager and Pioneer 10 spacecraft during the period 1977-1985 to measure the energy spectrum of oxygen with 4-125 MeV/nucleon, with particular emphasis on the energy interval from $\sim 4$ to $30 \mathrm{MeV} /$ nucleon, where the "anomalous" component of cosmic rays is dominant. This time period includes the sunspot minimum period at the beginning of solar cycle 21 , the time of the solar magnetic field reversal in 1980, the maximum solar modulation period of 1981-1983, and part of the recovery of the solar cycle through early 1985 . We find that the spectrum of anomalous oxygen changes dramatically after the middle of 1980 , with the peak or plateau region of the differential spectrum shifting to a higher energy. This change does not appear to be due to the increasing radial distance of the spacecraft in this time period, nor does it appear to be simply correlated with the level of modulation. The energy shift also appears to be significantly greater in the ecliptic plane than at $24^{\circ}$ north latitude (the heliographic position of Voyager 1 at the end of the time period).
\end{abstract}

\section{INTRODUCTION}

The study of the anomalous component of cosmic rays over the solar cycle may prove to be key to the understanding of the role of drifts in cosmic ray modulation. First observed in 1972 for helium [Garcia-Munoz et al., 1973] and oxygen [McDonald et al., 1974; Hovestadt et al., 1973], a total of four elements, $\mathrm{He}, \mathrm{N}, \mathrm{O}$, and $\mathrm{Ne}$, have now been observed to have anomalous spectra at low energies. These four elements all have high first ionization potentials, which led to the suggestion by Fisk et al. [1974] that the particles are interstellar neutrals which become singly ionized in the inner heliosphere and are subsequently accelerated to the $>5-\mathrm{MeV} /$ nucleon energy interval where they are observed.

Pesses et al. [1981] have suggested that the acceleration site of these particles is most likely in the polar regions of the solar wind termination shock. In their model the particles drift latitudinally toward the neutral sheet from the polar regions during periods of positive northern magnetic polarity but drift radially inward along the neutral sheet during the current period of negative northern polarity (after 1980). Consequently, they predict a strong dependence of the intensity of the anomalous component near the solar equator on solar magnetic field polarity.

In this analysis we make use of measurements of the spectrum of oxygen from the Cosmic Ray Subsystem (CRS) (see Stone et al., [1977] for a description of this experiment) on the Voyager 1 and 2 spacecraft and from the Goddard-University of New Hampshire experiment [McDonald et al., 1977] on the Pioneer 10 spacecraft for six quiet time intervals from 1977 to 1985. The positions of these spacecraft during the period make it possible to examine the spatial and temporal dependence of the observed variations and to address whether or not the anomalous oxygen spectrum exhibits changes associated with the polarity of the solar magnetic field.

Copyright 1986 by the American Geophysical Union.

Paper number 5A8657.

$0148-0227 / 86 / 005 \mathrm{~A}-8657 \$ 05.00$

\section{Observations}

The general features of the 11-year cosmic ray modulation cycle may be studied using the counting rate of the particles which penetrate the high-energy telescope on Voyager 1 . This rate is shown in Figure 1 for the year 1977-1985 and is mainly due to protons with $>75-\mathrm{MeV}$ energy. Note that the launch of Voyager 1 (and Voyager 2 as well) occurred near the end of the last solar minimum period when the particle tensity is at a maximum. The rate decreases beginning in 1978 and reaches a minimum in early 1981 and again in late 1982 . The current recovery of the intensity has not yet reached the levels of 1977 . In order to minimize the contamination by solar and interplanetary energetic particles, quiet times were selected by setting limits to the maximum low-energy helium flux, in a manner similar to that described by Cummings et al. [1984]. The six quiet time periods that were selected for analysis for Voyager 1 are shown as horizontal bars in Figure 1 and are labeled A-F. Also shown are the approximate times of the reversal of the solar polar magnetic fields [Webb et al., 1984]. Note that the field reversal occurs approximately between periods $C$ and $D$. In Table 1 we list the spacecraft positions in heliographic coordinates for the six quiet time periods. Note that Voyager 2 and Pioneer 10 are essentially in the ecliptic plane throughout the time period, whereas Voyager 1 leaves the ecliptic plane in 1980 (at the time of encounter with Saturn) and has an average latitude of $24^{\circ}$ for interval $F$.

The CRS telescopes, as well as the one on Pioneer 10, consist of an array of silicon solid-state detectors which measure the energy loss of nuclei that slow down and stop in the telescope. The technique for identification of the charge and energy of the particles is described in detail for the Voyager CRS telescopes by Cook et al. [1984] and for the Pioneer 10 telescope by McDonald et al. [1977].

The anomalous oxygen spectrum for the Voyager measurements is derived from the observed total oxygen spectrum for a particular time interval by subtracting a low-energy interplanetary component with a very steep spectrum and the highenergy galactic cosmic ray component. This subtraction procedure is illustrated in Figure 2 for two sample intervals. The 


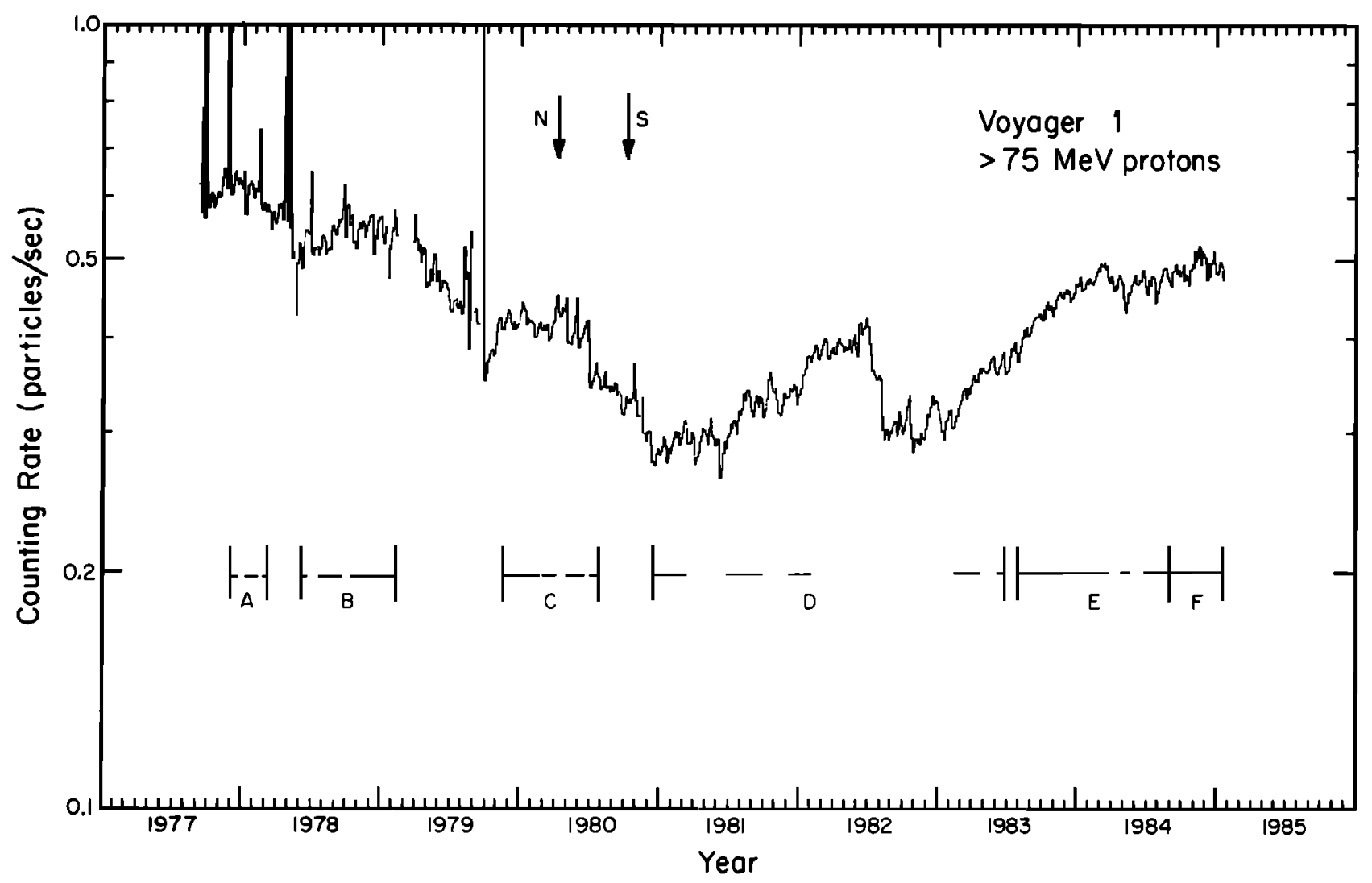

Fig. 1. Three-day average counting rate of penetrating particles in the HET 1 (high-energy telescope number 1) of the Voyager 1 CRS instrument from 1977 to 1986 . The rate is dominated by protons with energy $>75 \mathrm{MeV}$. The horizontal bars labeled A, B, C, D, E, and F refer to time intervals selected for construction of quiet time spectra of anomalous oxygen. The bars are discontinuous, thus reflecting the deletion of nonquiet times in the intervals. Similar intervals were also selected for Voyager 2 and Pioneer 10. The vertical arrows indicate approximate times of the solar magnetic field reversal at latitudes greater than $70^{\circ}$ in the north (N) and south (S).

TABLE 1. Average Heliographic Coordinates of Voyager 1, Voyager 2, and Pioneer 10 for Each of the Six Quiet Time Intervals

\begin{tabular}{|c|c|c|c|c|}
\hline Spacecraft & $\begin{array}{c}\text { Time } \\
\text { (Year/Day) }\end{array}$ & $\begin{array}{c}\text { Radial } \\
\text { Distance, } \\
\text { AU }\end{array}$ & $\begin{array}{l}\text { Latitude, } \\
\text { deg }\end{array}$ & $\begin{array}{c}\text { Longitude, } \\
\text { deg }\end{array}$ \\
\hline \multicolumn{5}{|c|}{ Interval $A$} \\
\hline V1 & $1977 / 337-1978 / 65$ & 2.1 & 0.5 & 185 \\
\hline V2 & $1977 / 337-1978 / 44$ & 2.0 & 4.1 & 183 \\
\hline P10 & $1977 / 1-1978 / 77$ & 13.6 & 6.4 & 159 \\
\hline \multicolumn{5}{|c|}{ Interval $B$} \\
\hline V1 & $1978 / 160-1979 / 40$ & 4.4 & -4.3 & 224 \\
\hline V2 & $1978 / 162-1979 / 35$ & 4.0 & -3.0 & 225 \\
\hline P10 & $1978 / 157-1979 / 287$ & 18.0 & 5.5 & 161 \\
\hline \multicolumn{5}{|c|}{ Interval C } \\
\hline V1 & $1979 / 324-1980 / 202$ & 7.5 & -5.2 & 272 \\
\hline V2 & $1979 / 306-1980 / 197$ & 6.4 & -5.5 & 265 \\
\hline P10 & $1980 / 1-1980 / 289$ & 21.7 & 5.0 & 166 \\
\hline \multicolumn{5}{|c|}{ Interval D } \\
\hline V1 & $1980 / 350-1983 / 174$ & 12.3 & 8.1 & 303 \\
\hline V2 & $1980 / 353-1983 / 175$ & 9.6 & -3.7 & 304 \\
\hline P10 & $1981 / 1-1983 / 182$ & 26.8 & 4.5 & 170 \\
\hline \multicolumn{5}{|c|}{ Interval $E$} \\
\hline V1 & $1983 / 207-1984 / 240$ & 18.6 & 21.9 & 323 \\
\hline v2 & $1983 / 225-1984 / 215$ & 13.4 & -1.2 & 344 \\
\hline P10 & $1983 / 261-1984 / 262$ & 32.4 & 4.0 & 173 \\
\hline \multicolumn{5}{|c|}{ Interval $F$} \\
\hline V1 & $1984 / 240-1985 / 16$ & 21.4 & 24.4 & 328 \\
\hline V2 & $1984 / 215-1984 / 334$ & 15.2 & -0.5 & 352 \\
\hline P10 & $1984 / 262-1985 / 17$ & 34.2 & 3.9 & 174 \\
\hline
\end{tabular}

Here V1, Voyager 1; V2, Voyager 2; P10, Pioneer 10. low-energy component is scaled and extrapolated from a power law fit to the observed helium spectrum in the same time interval in the energy range 3-6.1 MeV/nucleon using a ratio $\mathrm{He} / \mathrm{O}=100$. In calculating the uncertainty in the anomalous oxygen spectrum it was assumed that the uncertainty in the $\mathrm{He} / \mathrm{O}$ ratio was $\pm 50 \%$, which covers the range of compositions observed for corotating streams $(\mathrm{He} / \mathrm{O} \sim 150)$ and for solar flares $(\mathrm{He} / \mathrm{O} \sim 70)$ [Gloeckler et al., 1979]. The estimated galactic cosmic ray oxygen component is scaled from the observed carbon spectrum in the following way. The average combined carbon spectrum from Voyager 1 and 2 for each time interval is first fit to a power law from 20 to $106 \mathrm{MeV} /$ nucleon to determine the slope of the galactic cosmic ray component. Then the overall level is adjusted individually for Voyager 1 and 2 by a least squares fit to the observed oxygen intensity in the $66-$ to $125-\mathrm{MeV} /$ nucleon range. Based on the uncertainties derived in the fitting process the estimated uncertainty used in calculating the anomalous oxygen spectrum is $\pm 20 \%$.

In Figure $2 a$ it is evident that the spectrum of anomalous oxygen (shown by the open squares) from Voyager 1 for interval $A$ is almost identical to the observed spectrum in the energy range from 4 to $30 \mathrm{MeV} /$ nucleon. This is a time interval during solar minimum when the anomalous oxygen intensity is at its peak and the corrections for the solar and galactic cosmic ray components are essentially negligible. Figure $2 b$ depicts the spectrum from Voyager 2 for interval $D$, when the anomalous intensity is at a minimum and the corrections are much more significant.

Figure 3 shows the spectra of anomalous oxygen for the six 

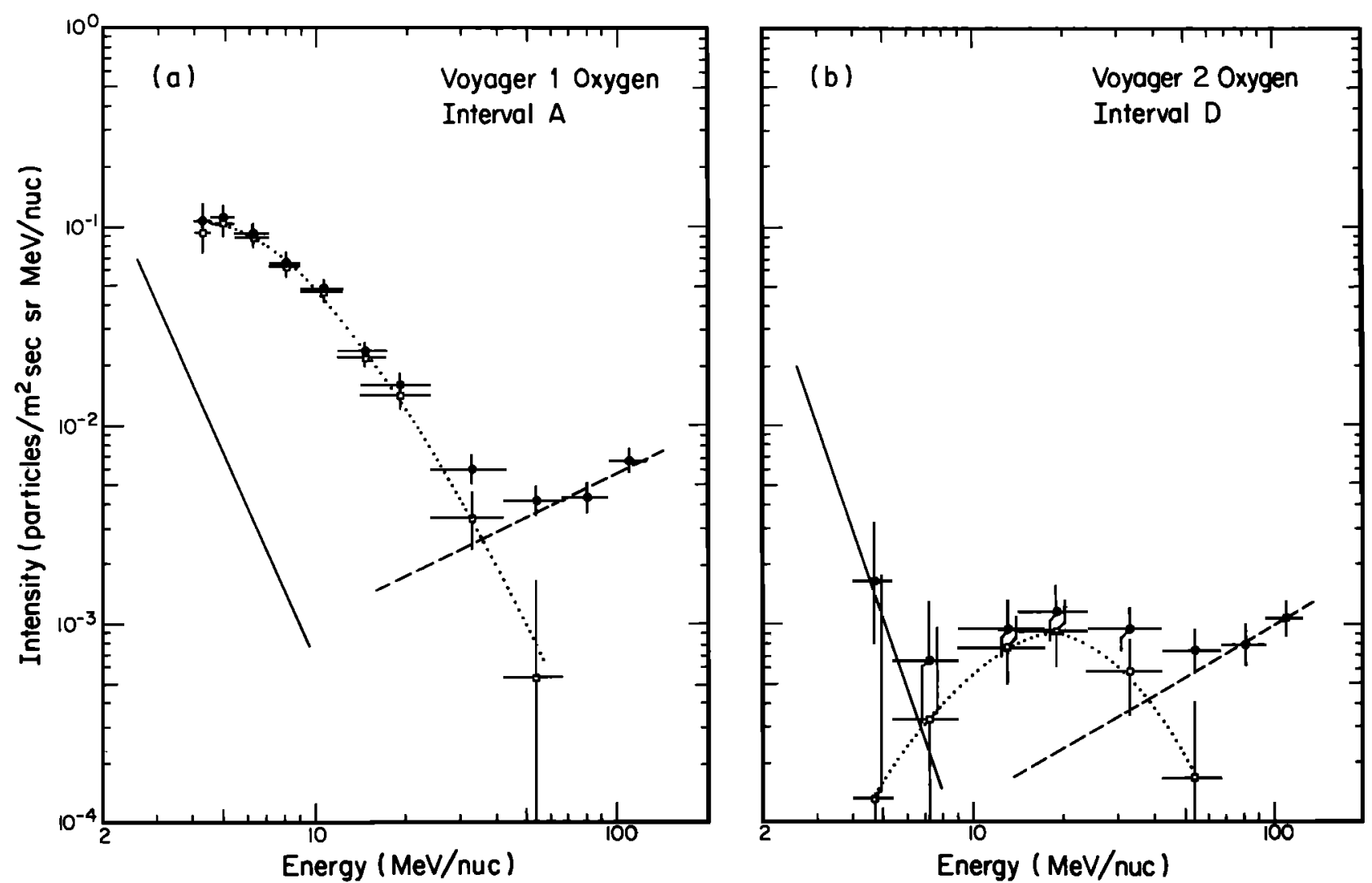

Fig. 2. Quiet time energy spectra of oxygen from Voyager data for two sample time intervals. The observed spectra are shown as solid circles. Estimated spectra of interplanetary and galactic components are shown as solid and dashed lines, respectively. The spectra of anomalous oxygen are indicated by the open squares which are joined by the dotted lines.
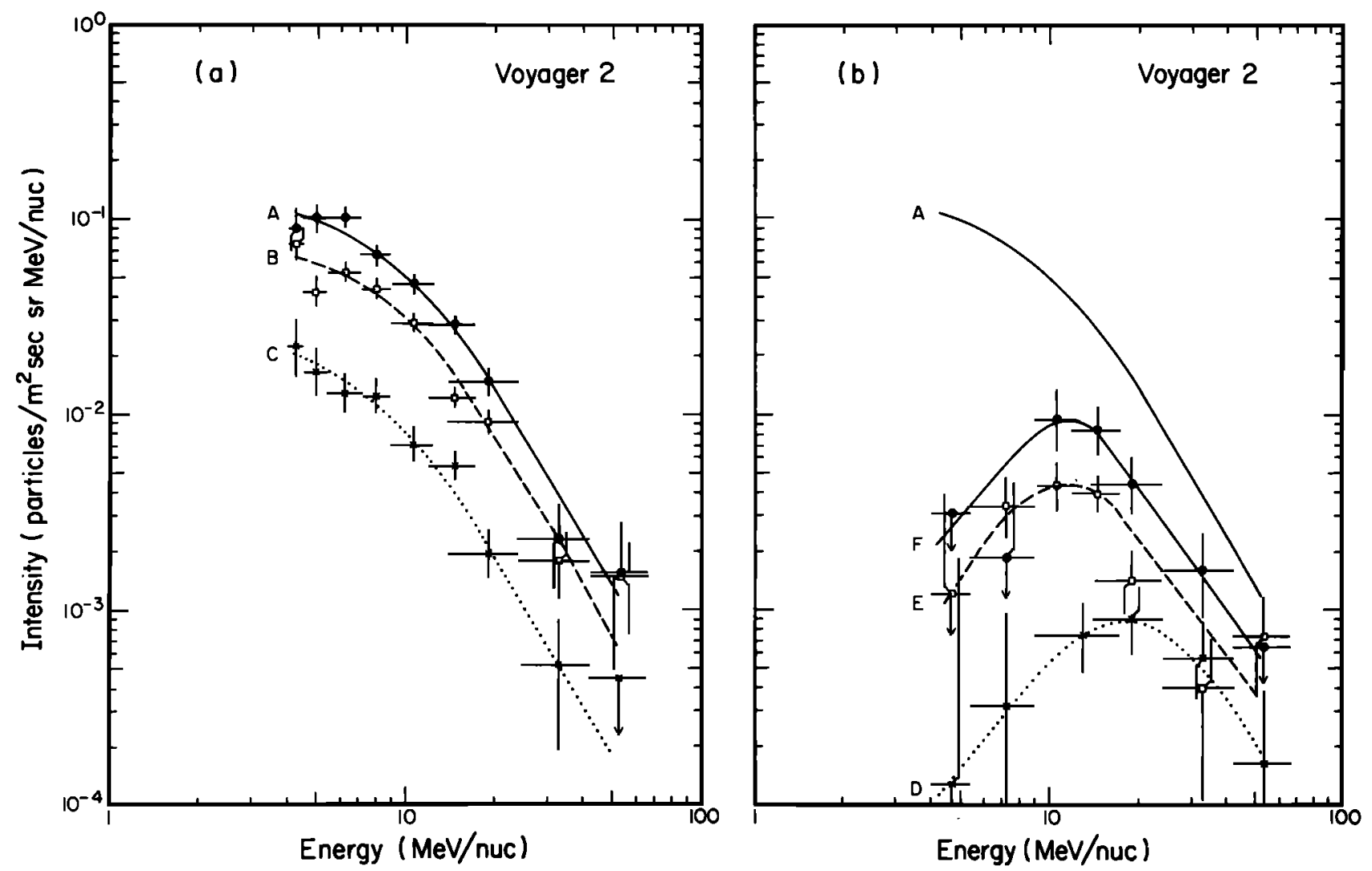

Fig. 3. Spectra of anomalous oxygen from Voyager 2 for the six time intervals described in Table 1. Figures $3 a$ and $3 b$ show the spectra for periods before and after the solar magnetic field reversal, respectively. The smooth curves are drawn to aide the eye and are not functional fits to the data. The solid curve labeled " $\mathrm{A}$ " in Figure $3 b$ is copied from Figure $3 a$ to facilitate comparison. Note the higher energy of the peak (or plateau) intensity of the spectra in Figure $3 b$ when compared to those of Figure $3 a$. 

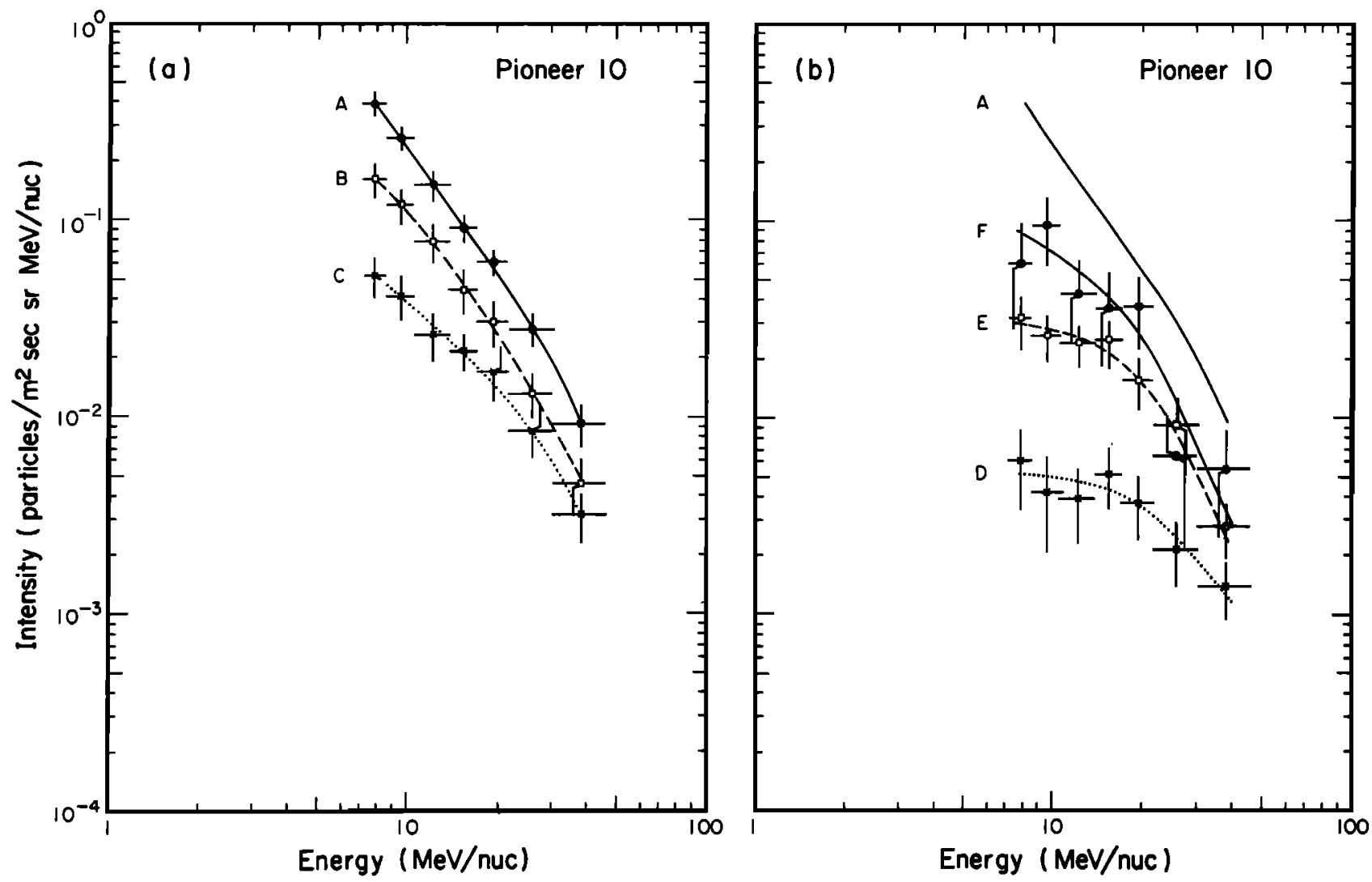

Fig. 4. Spectra of observed oxygen from Pioneer 10 for the six time intervals described in Table 1 in a format identical to that of Figure 3.
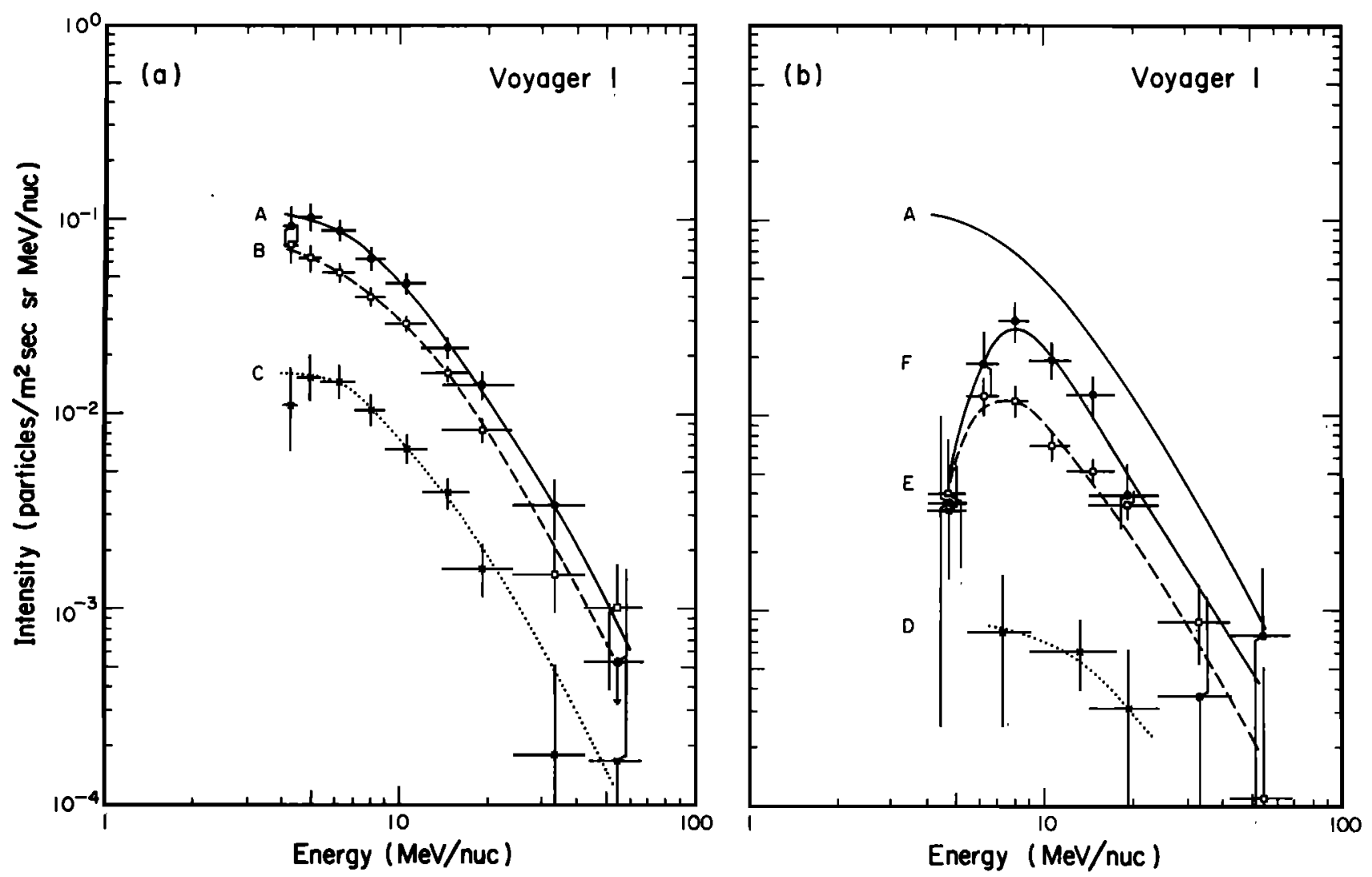

Fig. 5. Spectra of anomalous oxygen from Voyager 1 for the six time intervals described in Table 1 in a format identical to that of Figure 3. 


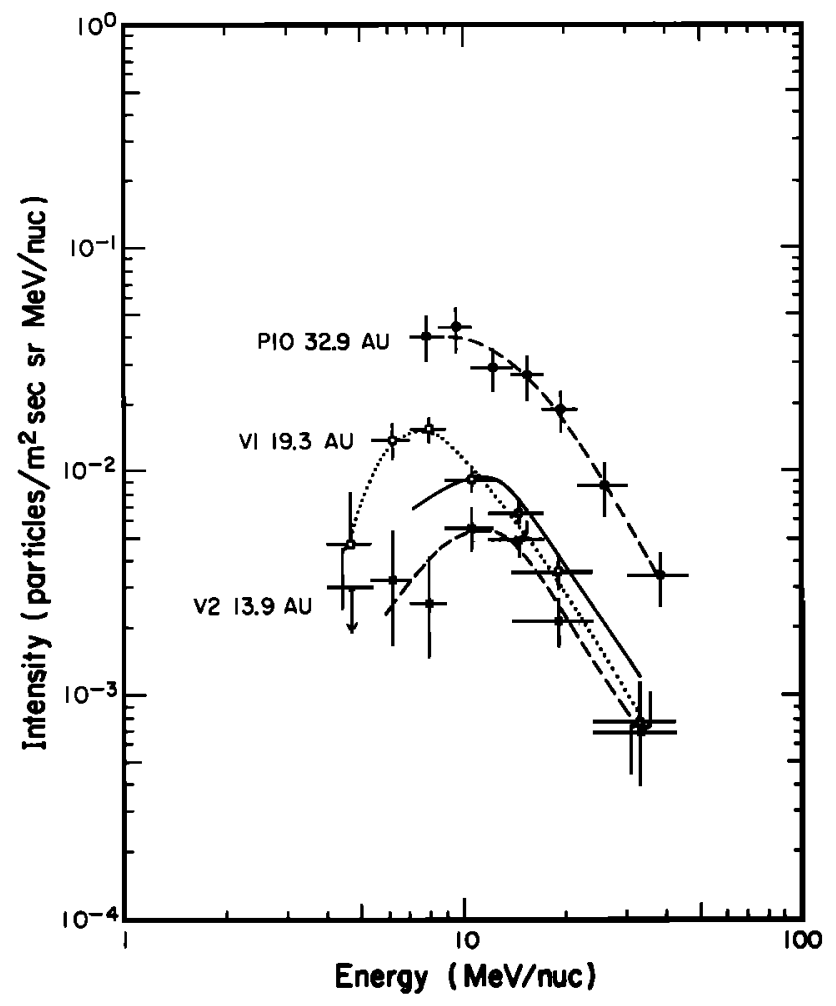

Fig. 6. Spectra of anomalous oxygen for the combined intervals $\mathrm{E}$ and F from Voyager 1 (V1) and Voyager (V2) and the spectrum of observed oxygen for the same combined intervals from Pioneer 10 (P10). The radial positions of the spacecraft in each time interval are shown. The solid curve represents the expected spectrum at $19.3 \mathrm{AU}$ in the ecliptic plane based on a radial gradient calculated as a function of energy from the two dashed curve approximations of the Voyager 2 and Pioneer 10 spectra. The lower peak energy for the Voyager 1 spectrum is apparently a result of its latitudinal position $\left(\sim 24^{\circ}\right)$.

quiet time intervals A-F derived from the Voyager 2 observations in the manner just described. Figures $3 a$ and $3 b$ show the spectra for the three intervals before and for the three intervals after the solar magnetic field reversal in 1980, respectively. The spectra for intervals A, B, and C are essentially monotonic, with a tendency to flatten into a peak or plateau at less than $\sim 6 \mathrm{MeV} /$ nucleon. It is clear from Figure $3 b$ that the energy dependence of the spectra for the intervals after the field reversal (D, E, and $F$ ) is significantly different, having a peak or plateau at $\sim 10-20 \mathrm{MeV} /$ nucleon.

The change in the energy spectrum of oxygen is also evident in the observed spectrum from the Pioneer 10 cosmic ray instrument. In Figure 4 we show these spectra for the same time intervals as those shown in Figure 3. Note that the intensities are considerably higher at Pioneer than at the Voyager spacecraft at comparable time intervals, and therefore it is not necessary to subtract the low-energy interplanetary and highenergy galactic components for the Pioneer data. Note the distinct flattening of the spectra in the $\sim 10$ - to 20 $\mathrm{MeV} /$ nucleon energy range after the mid-1980 period (Figure $4 b$ ) when compared to the earlier intervals shown in Figure 4a, indicating a change in the spectrum of anomalous oxygen similar to that of the Voyager 2 data.

In Figures $5 a$ and $5 b$ we show the spectra of anomalous oxygen derived from the Voyager 1 observations. In this case it is clear that the Voyager 1 spectra also show a shift of the peak energy location, but one that is not as large as that for the Voyager 2 and Pioneer 10 data. The peak in the spectra for intervals $\mathrm{E}$ and $\mathrm{F}$ in Figure $5 b$ appears at $\sim 8 \mathrm{MeV} / \mathrm{nu}-$ cleon, approximately one third lower than the corresponding peak energies for Voyager 2.

\section{Discussion}

The comparison of the two panels in each of the three figures discussed last (Figures 3, 4, and 5) suggests that the change in the energy spectrum of anomalous oxygen is correlated with the solar magnetic field reversal. However, all three spacecraft are moving outward from the sun, and Voyager 1 is moving out of the ecliptic plane as well (after late 1980), so that spatial variations must be considered in interpreting the data. In addition, the particle intensities are changing owing to modulation, and the peak energy location may be related to the level of modulation. We now investigate these possibilities.

We first address the difference in the energy dependence of Voyager 1 and 2 spectra. During the last two time intervals (E and F), Voyager 2, Voyager 1 , and Pioneer 10 have average radial distances from the sun of $13.9,19.3$, and $32.9 \mathrm{AU}$, respectively. Voyager 2 and Pioneer 10 are both in the ecliptic plane, whereas Voyager 1 is $\sim 24^{\circ}$ north in latitude. In Figure 6 we show the spectra of anomalous oxygen from Voyager 1 and 2 and the observed spectrum of oxygen from Pioneer 10 for the combined intervals $E$ and F. As noted earlier, the peak in the Voyager 1 spectrum is at a significantly lower energy than that of Voyager 2. We ask whether this effect could be simply due to the radial separation of the spacecraft. To address this question, we show in Figure 6 (solid curve) the calculated spectrum for $19.3 \mathrm{AU}$, the radial position of Voyager 1, using the radial gradient (logarithmic difference of particle intensities divided by the radial separation) inferred from the dashed curve approximations of the Pioneer 10 and Voyager 2 spectra. The resulting radial gradient varies slightly with energy, from $\sim 13 \% / \mathrm{AU}$ at $7 \mathrm{MeV} /$ nucleon to $\sim 10 \%$ / $\mathrm{AU}$ at $30 \mathrm{MeV} /$ nucleon. Above $\sim 10 \mathrm{MeV} /$ nucleon the predicted Voyager 1 spectrum and the observed spectrum are in reasonable agreement; however, the Voyager 1 anomalous oxygen flux in the $7.0-$ to $8.9-\mathrm{MeV} /$ nucleon energy range appears to be significantly higher than the prediction from radial gradient effects alone.

To quantify this difference, we have calculated the Voyager fluxes for the same energy intervals as those used in the Pioneer 10 spectrum. In Figure 7 we show the anomalous oxygen intensity as a function of radial distance from the sun for each of seven energy intervals for the combined time intervals $E$ and $F$. The slopes of the straight lines through the points at 13.9 and $32.9 \mathrm{AU}$ are the radial gradients based on the Voyager 2 and Pioneer 10 intensities. The points at $19.3 \mathrm{AU}$ are the Voyager 1 intensities. Above $10.6 \mathrm{MeV} /$ nucleon there is good agreement between the Voyager 1 intensities and the straight lines, indicating that at these energies the Voyager 1 intensities observed are those expected from a uniform radial gradient. At lower energies, however, the Voyager 1 intensities are significantly above the lines, indicating that in the 7.1- to 10.6-MeV/nucleon energy range, radial gradient effects alone cannot explain the Voyager 1 data. The vertical line at 19.3 $\mathrm{AU}$ on the line associated with the data in the 7.1- to 8.5 $\mathrm{MeV} /$ nucleon energy interval is the expected flux (68\% confidence interval) at the radial position of Voyager 1 if only radial effects are important. We find that the observed anomalous oxygen flux in this lowest energy interval is $\sim 4$ standard deviations above the expected value. Since Voyager 1 is at $\sim 24^{\circ}$ north latitude for this time interval, we suggest that this difference is due to the latitudinal separation of the spacecraft. In comparing the energy spectra of Voyager 1 and Voyager 2 in Figure 6 it appears that the peak of the energy spectrum of 


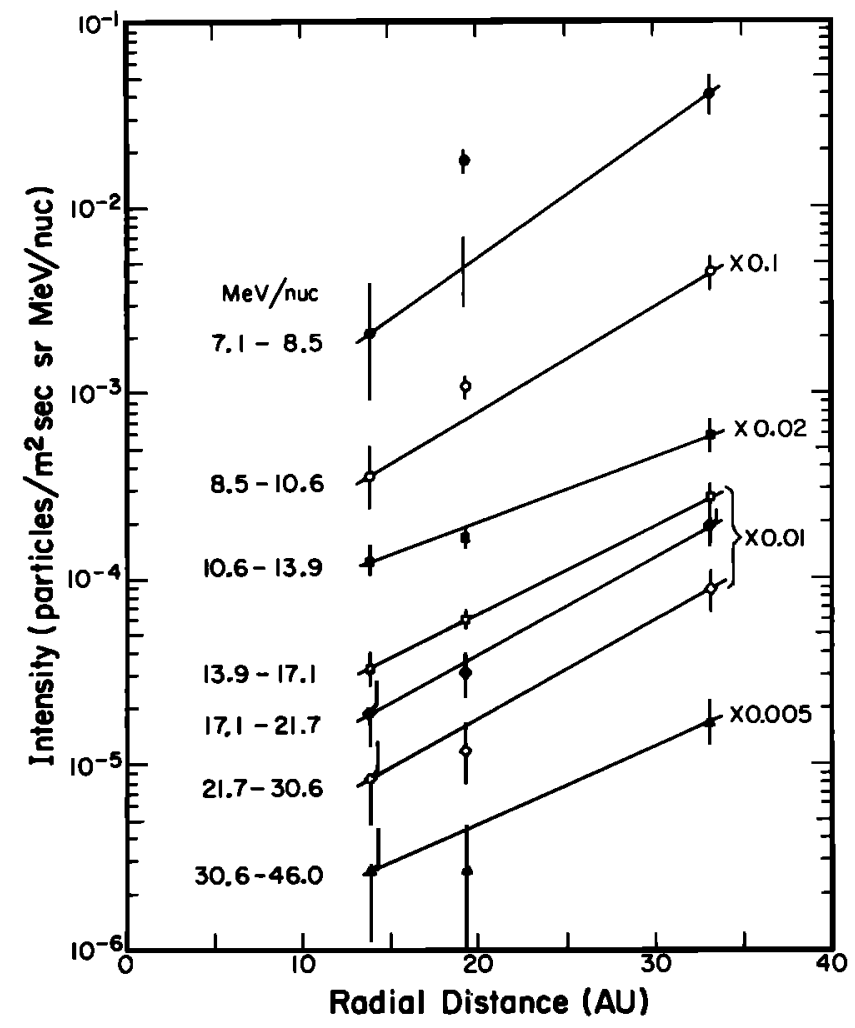

Fig. 7. Anomalous oxygen intensity for the combined time intervals $E$ and $F$ in seven energy intervals as measured on Pioneer 10 and the Voyager spacecraft. The points are plotted at the radial distances from the sun of the spacecraft. The intensities in the six energy intervals above $8.5 \mathrm{MeV} /$ nucleon have been multiplied by the factors shown to visually separate the data points. The lines are described in the text.

anomalous oxygen at such latitudes for this time interval is approximately one third lower than in the ecliptic plane.

To investigate the possibility that the peak energy location is correlated with the level of modulation, we show in Figure 8 the Voyager 1 observed oxygen spectra for intervals B and F. The galactic cosmic ray intensity is dominant in the 50- to $125-\mathrm{MeV} /$ nucleon energy range for both time intervals. The intensity in this energy range is only $\sim 20 \%$ higher for interval $B$ than for interval F, indicating that a similar level of modulation has been reached. Similarly, the anomalous oxygen intensity in the $10-$ to $30-\mathrm{MeV} /$ nucleon energy range is approximately the same for the two time intervals. However, below $10-\mathrm{MeV} /$ nucleon there is a factor of 10 difference in intensity, producing a striking difference in the spectrum. Because there is such a significant difference of the energy spectrum of anomalous oxygen in these two time intervals, we conclude that this spectral difference is not simply a result of the level of modulation of the particle intensities.

Since we have shown that a spatial effect, namely, a latitude variation, can account for the difference in the Voyager 1 and 2 spectra in the combined time interval $\mathrm{E}+\mathrm{F}$, it is reasonable to ask whether the increasing radial distance of the spacecraft could be responsible for the higher energy of the peak in the spectra seen in the ecliptic plane in intervals $D, E$, and $F$ as compared to intervals A, B, and C (see Figure 3). However, it is clear from a comparison of Figures $3 b$ and $4 a$ that radial position alone is not a key parameter in determining the peak energy, since Pioneer 10 and Voyager 2 were at similar radial distances and yet the peaks in the Pioneer 10 spectra are at a lower energy, $<7 \mathrm{MeV} /$ nucleon as opposed to $10-20 \mathrm{MeV} /$ nucleon. In addition, a comparison of the Pioneer 10 and Voyager 2 spectra in Figure 6, which are at the same time but at different radial distances, shows that the location of the peak is at the same energy or lower at Pioneer 10, not increasing with increasing radial distance as would be expected if the differences observed by Voyager 2 between intervals $\mathrm{A}-\mathrm{C}$ and D-F were due purely to a radial effect.

We conclude, therefore, that the changes in the energy dependence of the anomalous oxygen spectrum are not likely the result of either the level of modulation or radial position. Rather, the changes appear to be a temporal effect associated with the two halves of the solar cycle. If these changes are to be explained by "conventional" modulation theory, in which the effects of diffusion, convection, and adiabatic deceleration are considered and the effects of drifts ignored (see Fisk [1980] for a review of solar modulation theory), then it would require either a very prolonged "hysteresis" effect or a significant change in the rigidity dependence of the diffusion coefficient between halves of the solar cycle. A hysteresis effect (phase lag between intensity variations of low-rigidity and high-rigidity particles) has been reported for the anomalous oxygen component by Klecker et al. [1980] for the 1974-1975 period. They found a phase lag of 72 days between the intensity of anomalous oxygen with $7.6-24 \mathrm{MeV} /$ nucleon and the intensity of galactic cosmic rays with energy $>10 \mathrm{GeV}$. If the energy spectrum changes in our study are to be ascribed to hysteresis, then a phase lag of $\sim 4$ years would be required between particles with only modestly different rigidities and velocities. If changes in the diffusion coeflicient between halves of the solar cycle are to account for our observations, then the spectra of other cosmic ray species would show pronounced effects at the corresponding rigidities.

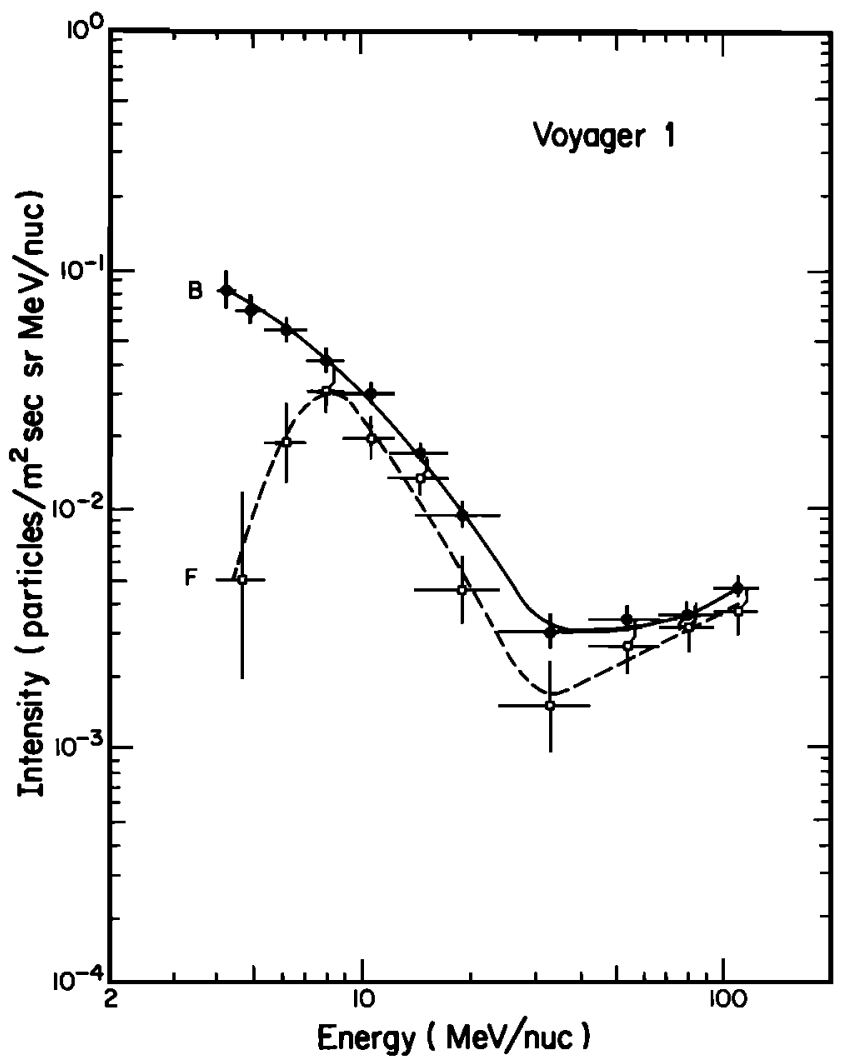

Fig. 8. Spectra of observed oxygen for time intervals $B$ and $F$ from Voyager 1 . Note the similarity in intensity level from $\sim 10$ to $125 \mathrm{MeV} /$ nucleon and the dramatic difference below $10 \mathrm{MeV} / \mathrm{nu}$ cleon. 
It appears more likely, therefore, that the observed time variation of the energy spectrum of anomalous oxygen is related to particle drift effects associated with the polarity of the solar magnetic field. Recently, J. R. Jokipii (private communication, 1985) has used a cosmic ray transport model which includes particle drifts and has calculated energy spectra of anomalous oxygen in the two halves of the solar cycle with different field polarity that resemble the observations reported here.

As we approach solar minimum, we plan further observations to extend these results to larger radial distances and higher latitudes and to include other species of the anomalous component, such as helium and nitrogen. These continued studies will permit a better characterization of the modulation of the anomalous component and improve our understanding of the role of drifts in cosmic ray propagation.

Acknowledgments. We are grateful to R. E. Vogt for his contributions as Principal Investigator for the Cosmic Ray Subsystem on the Voyager spacecraft. We also appreciate the contributions of the other CRS team members, consisting of scientists and engineers at the California Institute of Technology, the Goddard Space Flight Center, the University of Arizona, and the University of New Hampshire. This work was supported in part by NASA under contract NAS 7-918 and grant NGR 05-002-160.

The Editor thanks D. Hovestadt and another referee for their assistance in evaluating this paper.

\section{REFERENCES}

Cook, W. R., E. C. Stone, and R. Vogt, Elemental composition of solar energetic particles, Astrophys. J., 290, 827, 1984.

Cummings, A. C., E. C. Stone, and W. R. Webber, Evidence that the anomalous cosmic-ray component is singly ionized, Astrophys. $J$. Lett., 287, L99, 1984.

Fisk, L. A., Solar modulation of galactic cosmic rays, in Proceedings of the Conference on the Ancient Sun, edited by R. O. Pepin, J. A. Eddy, and R. B. Merrill, p. 103, Pergamon, New York, 1980.
Fisk, L. A., B. Kozlovsky, and R. Ramaty, An interpretation of the observed oxygen and nitrogen enhancements in low-energy cosmic rays, Astrophys. J. Lett., 190, L35, 1974.

Garcia-Munoz, M., G. M. Mason, and J. A. Simpson, A new test for solar modulation theory: The 1972 May-July low-energy galactic cosmic ray proton and helium spectra, Astrophys. J., 182, L81, 1973.

Gloeckler, G., D. Hovestadt, and L. A. Fisk, Observed distribution functions of $\mathrm{H}, \mathrm{He}, \mathrm{C}, \mathrm{O}$, and $\mathrm{Fe}$ in corotating energetic particle streams: Implications for interplanetary acceleration and propagation, Astrophys. J. Lett., 230, L191, 1979.

Hovestadt, D., O. Vollmer, G. Gloeckler, and C. Y. Fan, Differential energy spectra of low-energy ( $<8.5 \mathrm{MeV}$ per nucleon) heavy cosmic rays during solar quiet times, Phys. Rev. Lett., 31, 650, 1973.

Klecker, B., D. Hovestadt, G. Gloeckler, and C. Y. Fan, On the charge state of the anomalous oxygen component, Geophys. Res. Lett., 7, 1033, 1980.

McDonald, F. B., B. J. Teegarden, J. H. Trainor, and W. R. Webber, The anomalous abundance of cosmic-ray nitrogen and oxygen nuclei at low energies, Astrophys. J. Lett., 187, L105, 1974.

McDonald, F. B., N. Lal, J. H. Trainor, M. A. I. Van Hollebeke, and W. R. Webber, Observations of galactic cosmic-ray energy spectra between 1 and $9 \mathrm{AU}$, Astrophys. $J ., 216,930,1977$.

Pesses, M. E., J. R. Jokipii, and D. Eichler, Cosmic ray drift, shock wave acceleration, and the anomalous component of cosmic rays, Astrophys. J. Lett., 246, L85, 1981.

Stone, E. C., R. E. Vogt, F. B. McDonald, B. J. Teegarden, J. H. Trainor, J. R. Jokipii, and W. R. Webber, Cosmic ray investigation for the Voyager missions: Energetic particle studies in the outer heliosphere and beyond, Space Sci. Rev., 21, 355, 1977.

Webb, D. F., J. M. Davis, and P. S. McIntosh, Observations of the reappearance of polar coronal holes and the reversal of the polar magnetic field, Solar Phys., 92, 109, 1984.

A. C. Cummings and E. C. Stone, 220-47 Downs Laboratory, California Institute of Technology, Pasadena, CA 91125.

W. R. Webber, Space Sciences Center, DeMeritt Hall, University of New Hampshire, Durham, NH 03824.

(Received May 8, 1985; revised July 1, 1985 accepted July 2,1985 .) 